BMJ Open

Diabetes

Research

\& Care

\title{
Plasma phenylalanine and tyrosine and their interactions with diabetic nephropathy for risk of diabetic retinopathy in type 2 diabetes
}

\author{
Hui-Huan Luo, ${ }^{1,2}$ Juan Li, ${ }^{3}$ Xiao-Fei Feng, ${ }^{1}$ Xiao-Yu Sun, ${ }^{2}$ Jing Li, ${ }^{4}$ Xilin Yang,,${ }^{4,5}$ \\ Zhong-Ze Fang (D) 1,2,5
}

To cite: Luo H-H, Li J, Feng X-F, et al. Plasma phenylalanine and tyrosine and their interactions with diabetic nephropathy for risk of diabetic retinopathy in type 2 diabetes. BMJ Open Diab Res Care 2020;8:e000877. doi:10.1136/ bmjdrc-2019-000877

- Additional material is published online only. To view, please visit the journal online (http://dx.doi.org/10.1136/ bmjdrc-2019-000877).

H-HL and JuL contributed equally.

Received 3 September 2019 Revised 29 February 2020 Accepted 19 June 2020

\section{Check for updates}

(c) Author(s) (or their employer(s)) 2020. Re-use permitted under CC BY-NC. No commercial re-use. See rights and permissions. Published by BMJ.

For numbered affiliations see end of article.

Correspondence to Dr Zhong-Ze Fang;

fangzhongze@tmu.edu.cn and Dr Xilin Yang; yxl@hotmail.com

\section{ABSTRACT}

Objective Tight control of hyperglycemia reduces risk of diabetic retinopathy (DR), but the residual risk remains high. This study aimed to explore relationships between plasma phenylalanine and tyrosine with DR in type 2 diabetes (T2D) and interactions between the two amino acids, and their secondary interaction with renal dysfunction.

Research design and methods We extracted data of 1032 patients with T2D from tertiary hospital consecutively from May 2015 to August 2016. Binary logistic regression models with restricted cubic spline were used to check potential non-linear associations and to obtain ORs and $95 \% \mathrm{Cls}$ of variables under study. Addictive interaction was estimated using relative excess risk due to interaction, attributable proportion due to interaction and synergy index. Area under the receiver operating characteristic curve was used to check increased predictive values. Results 0 1032 patients, 162 suffered from DR. Copresence of low phenylalanine and low tyrosine increased DR risk (OR $6.01,95 \% \mathrm{Cl} 1.35$ to 26.8 ), while either of them alone did not have a significant effect with significant additive interaction. Presence of diabetic nephropathy further increased the OR of copresence of low phenylalanine and low tyrosine for DR to 25.9 (95\% $\mathrm{Cl} 8.71$ to 76.9 ) with a significant additive interaction. Inclusion of phenylalanine and tyrosine in a traditional risk factor model significantly increased area under the curve from 0.81 to $0.83(95 \% \mathrm{Cl} 0.80$ to 0.86$)$.

Conclusion Plasma low phenylalanine and low tyrosine worked independently and synergistically to increase the risk of DR in T2D. Presence of renal dysfunction further amplified the effect of copresence of low phenylalanine and low tyrosine on DR risk.

\section{INTRODUCTION}

Diabetic retinopathy (DR) is one of the major microvascular complications of diabetes, with about $30 \%$ prevalence, and leads to $5 \%-7 \%$ vision loss in Chinese diabetics. ${ }^{12}$ Tight glycaemia and blood pressure control are proven to reduce risk of DR and its progression; laser photocoagulation is the primary treatment for advanced DR and macular edema; new agents targeting vascular

\section{Significance of this study}

What is already known about this subject?

- Risk of diabetic retinopathy (DR) was still substantial despite tight hyperglycemia control. Dopamine (DA)-induced neurodegeneration and diabetic nephropathy (DN) were two risk factors for DR. Plasma phenylalanine and tyrosine were precursors of DA and were influenced by renal impairment.

- Whether plasma phenylalanine and tyrosine were related to DR development in type 2 diabetes (T2D) was uncertain; whether interactions between the two amino acids and their secondary interaction with renal dysfunction existed has not been investigated yet.

What are the new findings?

- In this hospital-based cross-sectional study, we found plasma low phenylalanine and low tyrosine increased the risk of DR in T2D with a significant addictive interaction.

- The effect of copresence of low phenylalanine and low tyrosine on DR risk was further amplified in patients with T2D with renal dysfunction.

- Inclusion of phenylalanine and tyrosine in the model increased predictive value beyond traditional risk factors.

How might these results change the focus of research or clinical practice?

- Our data suggested a new window for DR treatment, that is, modification of tyrosine/phenylalanine metabolism.

- In patients with diabetes with DN, control of tyrosine/ phenylalanine metabolism may confer great risk reduction for DR.

- More mechanism research was need to explore the role of abnormal tyrosine/phenylalanine metabolism in $\mathrm{DR}$, especially under the context of DN.

- More perspective epidemiology studies were warranted to confirm our findings in diverse races.

endothelial growth factor are under investigation. ${ }^{3}$ In spite of the continuous effort to reduce the burden of DR in diabetes, 
substantial residual risk is still high, and advanced DR is considered irreversible. There is a pressing need for new screening and treatments to prevent DR and DR-associated blindness.

Retinal neurodegeneration has been suggested to be an early event in the pathogenesis of DR and has been recognized as a new therapeutic perspective. ${ }^{4}$ Thus, identification of mediators in the crosstalk between neurodegeneration and DR is useful to further reduce DR burden in diabetes. Previous experiments showed that dopamine (DA) deficiency promoted early retinal dysfunction through neurodegeneration in diabetes rats. ${ }^{56} \mathrm{DA}$ is a catecholamine and exists in the retina and brain. It is derived from L-dihydroxyphenylalanine (L-DOPA), a hydroxylation product of tyrosine or phenylalanine when tyrosine was insufficient. ${ }^{7}$ Tyrosine and phenylalanine metabolism were perturbed in obesity, insulin resistance and pre-diabetes. ${ }^{8}$ It can be assumed that availability of amino acids, DA precursors, in blood regulates DA synthesis. In this connection, animal studies have shown that injecting tyrosine into rat brain stimulated dihydroxyphenylalanine synthesis. ${ }^{9}$ A previous prospective study has reported that decreased plasma tyrosine levels in diabetes are associated with increased risk of microvascular diseases. ${ }^{10}$ However, it remains unaddressed whether phenylalanine is also associated with risk of DR in diabetes.

Diabetic nephropathy (DN) is another primary microvascular complication of type 2 diabetes (T2D) and represents generalized endothelial dysfunction. ${ }^{11} \mathrm{DN}$ is associated with the presence of DR across diverse ethnicities through various common mechanisms. ${ }^{12}$ What's more, microvascular impairment and neurodegeneration could mutually promote each other and aggravate retinal injury. ${ }^{42}$ Impaired conversion of phenylalanine to tyrosine has been reported in renal impairment individuals. ${ }^{1013}$ Given the intimate relationships between tyrosine, phenylalanine, DN and DR, we ask whether there are biological interactions between tyrosine, phenylalanine and DN with increased risk of DR progression.

In the current cross-sectional study, we aimed to (1) test the associations between plasma levels of tyrosine, phenylalanine and risk of DR, and (2) explore possible interactions among tyrosine, phenylalanine and $\mathrm{DN}$ for the risk of DR in diabetes.

\section{MATERIALS AND METHODS}

\section{Research design and study patients}

The study patients and methods were described previously. ${ }^{14}$ Briefly, from May 2015 to August 2016, a total of 1898 consecutive inpatients of Liaoning Medical University First Affiliated Hospital were diagnosed with T2D. T2D was diagnosed by the 1999 WHO's criteria ${ }^{15}$ or treated with antidiabetic drugs. Electronic medical records were retrieved and metabolomics profiles were measured. A total of 1032 patients over 18 years old with complete information on age, height and weight were included in the current analysis.

\section{Data collection and definitions}

Information on demographic, anthropometric, current status of smoking and alcohol consumption, duration of diabetes, clinical and laboratory measurements, drugs used and disease status was recorded in electronic medical records. The clinical parameters included systolic blood pressure (SBP), diastolic blood pressure (DBP), triglyceride (TG), high-density lipoprotein cholesterol (HDL-C) and low-density lipoprotein cholesterol (LDLC), glycated hemoglobin (HbAlc) and serum creatinine (SCr). Medications included insulin, metformin and other antidiabetic agents, ACE inhibitors, angiotensin receptor blockers, and other antihypertensive drugs, statins and other lipid-lowering drugs. Disease status included DR, DN, coronary heart disease (CHD) and stroke.

\section{Clinical definitions}

Body mass index (BMI) was calculated by weight in kilogram divided by squared height in meter. The WHO criteria for Asians were used to classify BMI into four categories $^{16}$ : underweight at $<18.5 \mathrm{~kg} / \mathrm{m}^{2}$, normal weight at $18.5-24.0 \mathrm{~kg} / \mathrm{m}^{2}$, overweight at $24.0-28.0 \mathrm{~kg} / \mathrm{m}^{2}$ and obesity at $>28.0 \mathrm{~kg} / \mathrm{m}^{2}$. Hyperglycemia and abnormal lipids were defined as not reaching their treatment goals as recommended by the American Diabetes Association, ${ }^{17}$ that is, $\mathrm{HbAlc} \geq 7 \%$ for hyperglycemia, $\mathrm{TG} \geq 1.7 \mathrm{mmol} / \mathrm{L}$, LDL-C $\geq 2.6 \mathrm{mmol} / \mathrm{L}$ or HDL-C $\leq 1 \mathrm{mmol} / \mathrm{L}$ in men and HDL-C $\leq 1.3 \mathrm{mmol} / \mathrm{L}$ in women for abnormal lipids. The Cockcroft-Gault equation ${ }^{18}$ was used to obtain estimated glomerular filtration rate (eGFR) expressed in milliliters per minute per $1.73 \mathrm{~m}^{2}$ : eGFR $=[(140$-age $) \times-$ weight $\times 1.23 \times(0.85$ if female $) / \mathrm{SCr}]$.

DR was evaluated by the bilateral retinal photography and was defined as the presence, if any, of the following lesions: microaneurysms, retinal hemorrhages, soft exudates, hard exudates, or vitreous hemorrhage. DN was defined as persistent albuminuria, progressive reduction in glomerular filtration rate and hypertension judged by clinicians. CHD was defined as having a history of angina with abnormal ECG or on stress test, myocardial infarction, angina coronary artery bypass graft surgery or angioplasty; stroke was defined as nonfatal subarachnoid hemorrhage, intracerebral hemorrhage, or other unspecified intracranial hemorrhage and ischemic stroke.

\section{Laboratory assays}

Details of the amino acids measured were published previously. ${ }^{19}$ Briefly, liquid chromatography-mass spectrometry metabolomic analysis was conducted by using an AB Sciex 4000 QTrap system (AB Sciex, Framingham, Massachusetts, USA). Eight-hour fasting blood was taken and stored as dried blood spot. Each dried sample was dissolved in $100 \mu \mathrm{L}$ fresh mobile phase solution. The 
mobile phase was $80 \%$ acetonitrile aqueous solution. Amino acid quality control standards were provided by Chromsystems (Grafelfing, Germany). The standards were mixed and dissolved in $2 \mathrm{~mL}$ pure methanol and stored at $4^{\circ} \mathrm{C}$. Working solution was prepared through 100 -fold dilution for metabolite extraction. Analyst V.1.6.0 software (AB Sciex) was used for system control and data collection. ChemoView V.2.0.2 (AB Sciex) was used for data preprocessing.

\section{Statistical analysis}

Normally distributed continuous variables are reported as means (SD), and skewed variables are presented as medians (IQRs). Normality was checked by observing the $\mathrm{Q}-\mathrm{Q}$ plot. Categorical variables are presented as frequencies $(\%)$. The participants were divided into two groups by DR status. Differences between the two groups were compared by non-paired Student t-test for variables in normal distribution or Mann-Whitney $\mathrm{U}$ test for variables with a skewed distribution. The $\chi^{2}$ test (or Fisher test, if appropriate) was used to compare differences in categorical variables between the two groups. Binary logistic regression models were used to obtain ORs and 95\% CIs of the variables of interest for DR. Restricted cubic spline (RCS) nested in the logistic regression was used to check potential non-linear associations between amino acid levels with the risk of DR. In the analysis, three knots were chosen in RCS as suggested by Harrell. ${ }^{20}$ As before, ${ }^{21}$ cutoff points of phenylalanine and tyrosine for DR were selected at the points where the risk of DR started to rise rapidly. Apart from clinical characteristics, tyrosine and phenylalanine, we also depicted a more global amino acid profile briefly.

A structured adjustment scheme was used to control for confounding effects by other variables. First, we obtained the unadjusted OR. Second, multivariable analysis was performed to adjust for traditional risk factors, including age, sex, BMI and duration of diabetes (model 1 ); the variables in model 1 plus SBP, HDL-C, LDL-C, HbA1c and TG (model 2); the variables in model 2 plus antidiabetic drugs, lipid-lowering drugs and antihypertensive drugs (model 3); the variables in model 3 plus DN (model 4); and the variables in model 4 plus tyrosine and phenylalanine in a single model (model 5 ).

Additive interaction was used to check interactions between any pairs of tyrosine, phenylalanine and DN for DR. ${ }^{14}$ Relative excess risk due to interaction (RERI), attributable proportion (AP) due to interaction and synergy index (S) were used to estimate the interactions. RERI $>0, A P>0$ or $\mathrm{S}>1$ indicates a significant additive interaction or synergistic effect. Two levels of additive interactions were examined. First, we examined additive interactions between tyrosine and phenylalanine for DR. Then, we examined additive interactions between DN and copresence of low tyrosine and low phenylalanine as a second-order interaction. Variables listed in model 4 were adjusted in the first-order interaction analysis, and the variables listed model 3 were adjusted in the secondorder interaction analysis.

Areas under the receiver operating characteristic curves were compared with estimate the potential predictive value of tyrosine or/and phenylalanine beyond traditional risk factors for DR.

Plasma free amino acids levels could be affected by metabolic state, especially insulin resistance and glycemic control. So, to attenuate confounding effects due to likelihood of DR, we also obtained a subgroup with relevant balanced characteristics from the total cohort with propensity score matching. Propensity score was calculated using a logistic regression procedure with DR as dependent variable and age, sex, BMI, duration of diabetes, SBP, HDL-C, LDL-C, HbA1C, TG, DN, antidiabetic drugs, lipid-lowering drugs and antihypertensive drugs as independent variables. Differences of characteristics between patients with and without DR and OR of amino acids for DR risk were estimated.

$P$ values of $<0.05$ were considered statistically significant in all these analyses. All analyses were conducted using SAS V.9.4.

\section{RESULTS}

\section{Characteristics of the study patients}

The 1032 patients had a mean age of 57.2 (SD 13.8) years and A median duration of T2D of 5 (IQR 0-10) years. Of these patients, $53.2 \%$ were male. The mean BMI of the cohort was 25.3 (SD 3.9) $\mathrm{kg} / \mathrm{m}^{2}$, while $63.1 \%$ of the patients were overweight or obese. Of these patients, 162 were with prior DR, while 870 were not. There were 188 (18.2\%), $210(20.3 \%)$ and $199(19.3 \%)$ individuals with prior DN, CHD, and stroke, respectively. Subjects with DR composed more women and had longer duration of diabetes, higher SBP, more lack of HbAlc, HDL-C, LDL-C and TG, more insulin use, more DN, and less CHD and stroke compared with those without DR. Age, BMI, current smoking, current drinking, DBP, eGFR, creatinine, and drug use (except for insulin) were similar in the two groups. Tyrosine and phenylalanine were lower in DR then in non-DR (table 1).

\section{Associations of tyrosine and phenylalanine with DR risk}

Both tyrosine and phenylalanine were inversely associated with risk of DR in a non-linear manner (figure 1). Decreasing levels of tyrosine at $<64 \mu \mathrm{mol} / \mathrm{L}$ and phenylalanine at $<64 \mu \mathrm{mol} / \mathrm{L}$ were associated with a rapid rise in the risk of DR both in univariable (OR for tyrosine 5.11, 95\% CI 2.22 to 11.8 ; OR for phenylalanine 6.32 , 95\% CI 2.30 to 17.4 ) and multivariable analyses adjusted for traditional risk factors, that is, age, sex, BMI, duration of diabetes, SBP, HDL-C, LDL-C, HbA1C, TG, antidiabetic drugs, lipid-lowering drugs, antihypertensive drugs and DN (OR for tyrosine 4.06, 95\% CI 1.66 to 9.94; OR for phenylalanine: $7.57,95 \%$ CI 2.53 to 22.6) (table 2). When further adjusted for the other amino acid, the ORs were attenuated. 
Table 1 Characteristics of patients with type 2 diabetes according to DR status

\begin{tabular}{|c|c|c|c|c|}
\hline & Total & DR & Non-DR & $P$ value \\
\hline $\mathrm{N}$ & 1032 & 162 & 870 & \\
\hline Age (years) & $57.2(13.8)$ & $57.8(10.0)$ & $57.1(14.4)$ & 0.491 \\
\hline Sex, male & $549(53.2 \%)$ & $73(45.1 \%)$ & $476(54.7 \%)$ & 0.024 \\
\hline Duration of DM (years) & $5(0-10)$ & 13(6-20) & $4(0-10)$ & $<0.000$ \\
\hline Body mass index $\left(\mathrm{kg} / \mathrm{m}^{2}\right)$ & $25.3(3.9)$ & $25.1(3.3)$ & $25.3(3.9)$ & 0.371 \\
\hline$\sim 18.5$ & 27 (2.6\%) & $1(0.6 \%)$ & $26(3.0 \%)$ & 0.176 \\
\hline $18.5 \sim 24.0$ & $354(34.3 \%)$ & $64(39.5 \%)$ & $290(33.3 \%)$ & \\
\hline $24.0 \sim 28.0$ & $430(41.7 \%)$ & $66(40.7 \%)$ & $364(41.8 \%)$ & \\
\hline $28.0 \sim$ & $221(21.4 \%)$ & $31(19.1 \%)$ & $190(21.8 \%)$ & \\
\hline Current smoking & & $42(25.9 \%)$ & 289 (33.2\%) & 0.068 \\
\hline Current drinking & & $42(25.9 \%)$ & 248 (28.5\%) & 0.502 \\
\hline SBP (mm Hg) & & $145.6(25.3)$ & $139.4(23.6)$ & 0.003 \\
\hline DBP (mm Hg) & & $83.0(13.4)$ & $82.3(13.5)$ & 0.551 \\
\hline HbA1c (\%) & & $9.42(2.35)$ & $9.63(2.38)$ & 0.409 \\
\hline$\sim 7$ & & $15(9.3 \%)$ & $62(7.1 \%)$ & 0.582 \\
\hline $7 \sim 8$ & & $16(9.9 \%)$ & $90(10.3 \%)$ & \\
\hline $8 \sim$ & & 64 (39.5\%) & $384(44.1 \%)$ & \\
\hline Lack & & $67(41.4 \%)$ & $334(38.4 \%)$ & \\
\hline $\mathrm{HDL}-\mathrm{C}(\mathrm{mmol} / \mathrm{L})$ & & $1.04(0.87-1.32)$ & $1.01(0.85-1.25)$ & 0.254 \\
\hline$<1.00$ in men or $<1.30$ in women & & $36(22.2 \%)$ & $211(24.3 \%)$ & $<0.000$ \\
\hline$\geq 1.00$ in men or $\geq 1.30$ in women & & $58(35.8 \%)$ & $436(50.1 \%)$ & \\
\hline Lack & & $68(42.0 \%)$ & $223(25.6 \%)$ & \\
\hline LDL-C (mmol/L) & & $2.83(2.27-3.38)$ & $2.78(2.20-3.39)$ & 0.583 \\
\hline$<2.60$ & & $38(23.5 \%)$ & 269 (30.9\%) & 0.000 \\
\hline$\geq 2.60$ & & $56(34.6 \%)$ & $378(43.5 \%)$ & \\
\hline Lack & & $68(42.0 \%)$ & $223(25.6 \%)$ & \\
\hline Triglyceride (mmol/L) & & $1.72(1.13-2.48)$ & $1.66(1.11-2.37)$ & 0.544 \\
\hline$<1.70$ & & $46(28.4 \%)$ & $337(38.7 \%)$ & $<0.000$ \\
\hline$\geq 1.70$ & & $48(29.6 \%)$ & $313(36.0 \%)$ & \\
\hline Lack & & $68(42.0 \%)$ & $220(25.3 \%)$ & \\
\hline eGFR $\left(\mathrm{mL} / \mathrm{min} / 1.73 \mathrm{~m}^{2}\right)$ & & $109.7(48.4)$ & $112.9(51.6)$ & 0.495 \\
\hline$\sim 60$ & & $14(9.7 \%)$ & $101(13.7 \%)$ & 0.125 \\
\hline $60 \sim 90$ & & $40(27.6 \%)$ & $153(20.7 \%)$ & \\
\hline $90 \sim$ & & $91(62.8 \%)$ & $486(65.7 \%)$ & \\
\hline Serum creatinine ( $\mu \mathrm{mol} / \mathrm{L})$ & & 56.65 (48.77-72.49) & $60.22(49.39-74.53)$ & 0.314 \\
\hline Antidiabetic agents & & 137 (84.6\%) & $730(83.9 \%)$ & 0.907 \\
\hline Insulin & & $132(81.5 \%)$ & $640(73.6 \%)$ & 0.038 \\
\hline Metformin & & $51(31.5 \%)$ & $307(35.3 \%)$ & 0.370 \\
\hline Hypotensive agents & & $71(43.8 \%)$ & $342(39.3 \%)$ & 0.281 \\
\hline ACEI & & $23(14.2 \%)$ & $112(12.9 \%)$ & 0.614 \\
\hline ARB & & $26(16.1 \%)$ & $108(12.4 \%)$ & 0.205 \\
\hline$\beta$-blockers & & $8(4.9 \%)$ & $85(9.8 \%)$ & 0.052 \\
\hline Lipid-lowering agents & & $63(38.9 \%)$ & $325(37.4 \%)$ & 0.712 \\
\hline Statins & & $60(37.0 \%)$ & $310(35.6 \%)$ & 0.732 \\
\hline Other lipid-lowering agents & & $4(2.5 \%)$ & $19(2.2 \%)$ & 0.773 \\
\hline
\end{tabular}


Table 1 Continued

\begin{tabular}{|c|c|c|c|c|}
\hline & Total & DR & Non-DR & $P$ value \\
\hline Diabetic nephropathy & $188(18.2 \%)$ & $64(39.5 \%)$ & $124(14.3 \%)$ & $<0.000$ \\
\hline Coronary heart disease & $210(20.3 \%)$ & $23(14.2 \%)$ & $187(21.5 \%)$ & 0.034 \\
\hline Stroke & $199(19.3 \%)$ & $17(10.5 \%)$ & $182(20.9 \%)$ & 0.002 \\
\hline Tyrosine ( $\mu \mathrm{mol} / \mathrm{L})$ & $45.78(36.70-56.27)$ & $41.51(12.42)$ & $49.54(17.38)$ & $<0.000$ \\
\hline$<64$ & $883(85.6 \%)$ & $156(96.3 \%)$ & $727(83.6 \%)$ & $<0.000$ \\
\hline$\geq 64$ & $149(14.4 \%)$ & $6(3.7 \%)$ & $143(16.4 \%)$ & \\
\hline Phenylalanine $(\mu \mathrm{mol} / \mathrm{L})$ & $45.34(37.13-54.46)$ & $40.97(10.21)$ & $48.34(14.27)$ & $<0.000$ \\
\hline$<64$ & $908(88.0 \%)$ & $158(97.5 \%)$ & $750(86.2 \%)$ & $<0.000$ \\
\hline$\geq 64$ & $124(12.0 \%)$ & $4(2.5 \%)$ & $120(13.8 \%)$ & \\
\hline
\end{tabular}

Data are mean (SD), median (IQR), or $\mathrm{n}(\%)$.

$P$ value was acquired by comparing DR and non-DR; $p$ values were derived from independent-samples Student t-test for normally distributed variables, Mann-Whitney $U$ test for skewed distributions, and $\chi^{2}$ test (or Fisher test, if appropriate) for categorical variables. There were 401 (38.9\%), 291 (28.2\%), 291 (28.2\%), and 288 (27.9\%) missing values in HbA1c, HDL-C, LDL-C and triglyceride, respectively. ACEI, ACE inhibitor; ARB, angiotensin receptor blocker; DBP, diastolic blood pressure; DM, diabetes mellitus; DR, diabetic retinopathy; eGFR, estimated glomerular filtration rate; HbA1c, glycated hemoglobin; HDL-C, high-density lipoprotein cholesterol; LDL-C, low-density lipoprotein cholesterol; SBP, systolic blood pressure.

Values are ORs (95\% CIs) from logistic regression analyses. Model 1 was adjusted for age, sex, BMI $(<18.5$, $18.5-24.0,24.0-28.0$ and $\left.>28.0 \mathrm{~kg} / \mathrm{m}^{2}\right)$ and duration of diabetes. Model 2 was adjusted for variables in model one plus SBP, HDL-C $(<1.0 \mathrm{mmol} / \mathrm{L}$ in men or $<1.3 \mathrm{mmol} / \mathrm{L}$ in women, $\geq 1.0 \mathrm{mmol} / \mathrm{L}$ in men or $\geq 1.3 \mathrm{mmol} / \mathrm{L}$ in women, lack), LDL-C $(<2.6$ and $\geq 2.6 \mathrm{mmol} / \mathrm{L}$, lack $)$, HbAlc
(<7\%, 7\% 8\%, $\geq 8 \%$, lack), TG $(<1.7$ and $\geq 1.7 \mathrm{mmol} / \mathrm{L}$, lack). Model 3 was adjusted for variables in model 2 plus antidiabetic drugs, lipid-lowering drugs and antihypertensive drugs. Model 4 was adjusted for variables in model 3 plus DN. Model 5 was adjusted for variables in model 4 and Tyr or Phe, respectively. The concentration of Phe was coded to $45.34 \mu \mathrm{mol} / \mathrm{L}$ (mean) if Phe
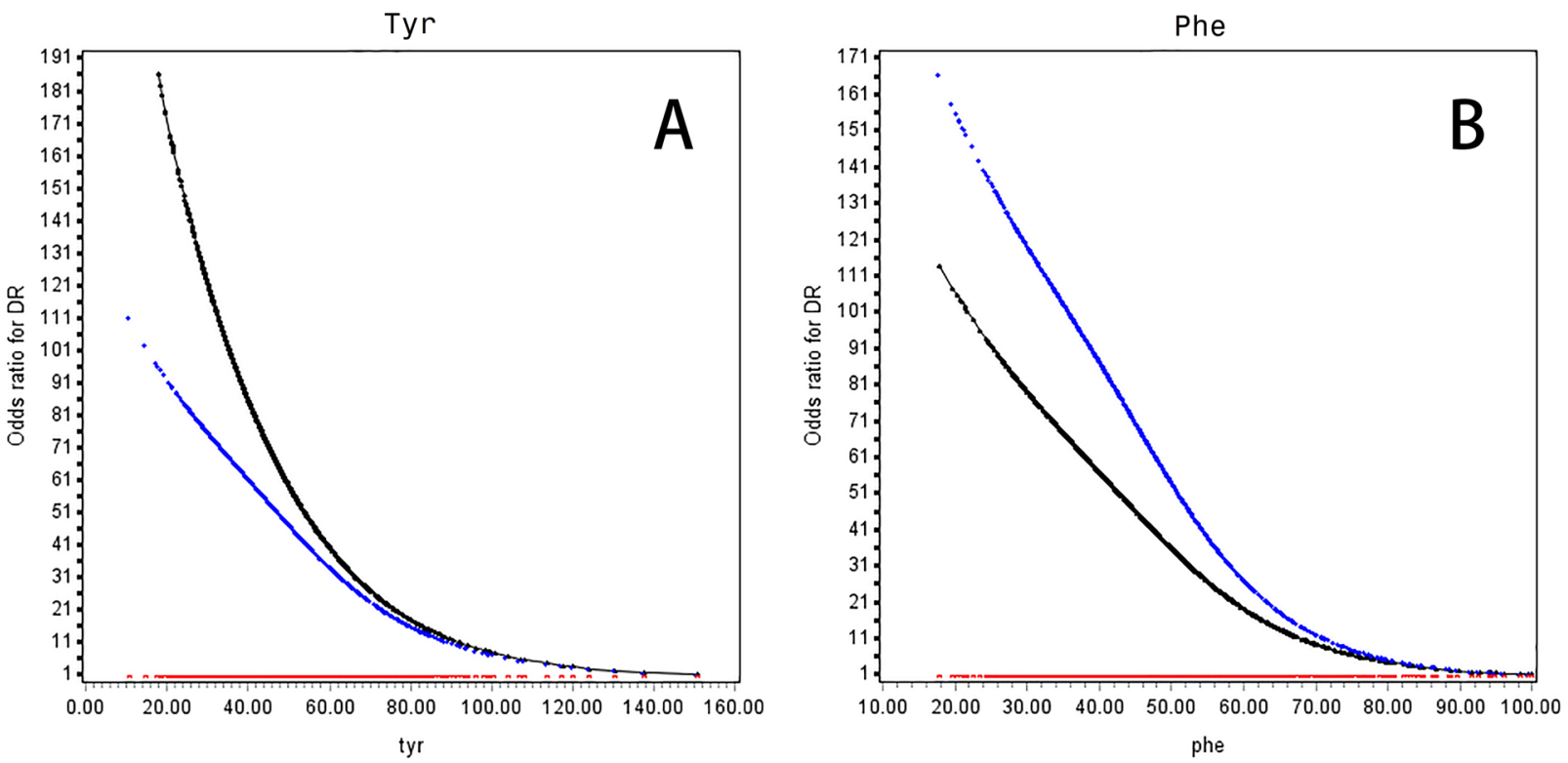

Figure 1 OR curves of Tyr (A) and Phe (B) for DR in Chinese patients with T2D. The black curve was derived from univariable analysis, and the blue curve was derived from multivariate analysis that adjusted for age, sex, body mass index $(<18.5$, $18.5-24.0,24.0-28.0$ and $>28.0 \mathrm{~kg} / \mathrm{m}^{2}$ ), duration of diabetes, systolic blood pressure, high-density lipoprotein cholesterol $(<1.0 \mathrm{mmol} / \mathrm{L}$ in men or $<1.3 \mathrm{mmol} / \mathrm{L}$ in women, $\geq 1 \mathrm{mmol} / \mathrm{L}$ in men or $\geq 1.3 \mathrm{mmol} / \mathrm{L}$ in women, lack), low-density lipoprotein cholesterol ( $<2.6$ and $\geq 2.6 \mathrm{mmol} / \mathrm{L}$, lack), $\mathrm{HbA} 1 \mathrm{c}(<7 \%, 7 \% \sim 8 \%$, and $\geq 8 \%$, lack), triglyceride $(<1.7$ and $\geq 1.7 \mathrm{mmol} / \mathrm{L}$, lack), diabetic nephropathy, antidiabetic drugs, lipid-lowering drugs and antihypertensive drugs. The red curve stands for the reference level (ie, the OR for DR was 1). The concentration of Phe was coded to $45.34 \mu \mathrm{mol} / \mathrm{L}$ (mean) if Phe $\geq 100.00 \mu \mathrm{mol} / \mathrm{L}$. Missing values of $\mathrm{HbA} 1 \mathrm{c}$ and lipids were presented as one category. DR, diabetic retinopathy; $\mathrm{HbA1c}$, glycated hemoglobin; Phe, phenylalanine; T2D, type 2 diabetes; Tyr, tyrosine. 


\begin{tabular}{|c|c|c|c|}
\hline & OR & $95 \% \mathrm{Cl}$ & $P$ value \\
\hline \multicolumn{4}{|l|}{ Univariable model } \\
\hline Tyr $<64$ vs $\geq 64(\mu \mathrm{mol} / \mathrm{L})$ & 5.11 & 2.22 to 11.8 & $<0.001$ \\
\hline Phe $<64$ vs $\geq 64(\mu \mathrm{mol} / \mathrm{L})$ & 6.32 & 2.30 to 17.4 & $<0.001$ \\
\hline \multicolumn{4}{|l|}{ Multivariable model 1} \\
\hline Tyr $<64$ vs $\geq 64(\mu \mathrm{mol} / \mathrm{L})$ & 4.21 & 1.79 to 9.93 & 0.001 \\
\hline Phe $<64$ vs $\geq 64(\mu \mathrm{mol} / \mathrm{L})$ & 7.34 & 2.57 to 21.0 & $<0.001$ \\
\hline \multicolumn{4}{|l|}{ Multivariable model 2} \\
\hline Tyr <64 vs $\geq 64(\mu \mathrm{mol} / \mathrm{L})$ & 4.25 & 1.79 to 10.1 & 0.001 \\
\hline Phe $<64$ vs $\geq 64(\mu \mathrm{mol} / \mathrm{L})$ & 6.99 & 2.45 to 19.99 & $<0.001$ \\
\hline \multicolumn{4}{|l|}{ Multivariable model 3} \\
\hline Tyr $<64$ vs $\geq 64(\mu \mathrm{mol} / \mathrm{L})$ & 4.18 & 1.75 to 9.99 & 0.001 \\
\hline Phe $<64$ vs $\geq 64(\mu \mathrm{mol} / \mathrm{L})$ & 7.18 & 2.49 to 20.7 & $<0.001$ \\
\hline \multicolumn{4}{|l|}{ Multivariable model 4} \\
\hline Tyr <64 vs $\geq 64(\mu \mathrm{mol} / \mathrm{L})$ & 4.06 & 1.66 to 9.94 & 0.002 \\
\hline Phe $<64$ vs $\geq 64(\mu \mathrm{mol} / \mathrm{L})$ & 7.57 & 2.53 to 22.6 & $<0.001$ \\
\hline \multicolumn{4}{|l|}{ Multivariable model 5} \\
\hline Tyr <64 vs $\geq 64(\mu \mathrm{mol} / \mathrm{L})$ & 2.75 & 1.11 to 6.83 & 0.029 \\
\hline Phe $<64$ vs $\geq 64(\mu \mathrm{mol} / \mathrm{L})$ & 5.83 & 1.92 to 17.7 & 0.002 \\
\hline
\end{tabular}

Values are OR $(95 \% \mathrm{Cls})$ from logistic regression analyses. Model 1 was adjusted for age, sex, body mass index $(<18.5$ $\mathrm{kg} \cdot \mathrm{m}^{2}, 18.5 \mathrm{~kg} \cdot \mathrm{m}^{2} 24.0 \mathrm{~kg} \cdot \mathrm{m}^{2}, 24.0 \mathrm{~kg} \cdot \mathrm{m}^{2}-28.0 \mathrm{~kg} \cdot \mathrm{m}^{2}$ and $>28.0$ $\mathrm{kg} \cdot \mathrm{m}^{2}$ ) and duration of diabetes. Model 2 was adjusted for variables in model 1 plus systolic blood pressure, high-density lipoprotein cholesterol $(<1.0 \mathrm{mmol} / \mathrm{L}$ in male or $<1.3 \mathrm{mmol} / \mathrm{L}$ in female, $\geq 1.0 \mathrm{mmol} / \mathrm{L}$ in male or $\geq 1.3 \mathrm{mmol} / \mathrm{L}$ in female, lack), lowdensity lipoprotein cholesterol $(<2.6 \mathrm{mmol} / \mathrm{L}, \geq 2.6 \mathrm{mmol} / \mathrm{L}$, lack), glycated hemoglobin $(<7 \%, 7 \% \sim 8 \%, \geq 8 \%$, lack), triglyceride $(<1.7 \mathrm{mmol} / \mathrm{L}, \geq 1.7 \mathrm{mmol} / \mathrm{L}$, lack). Model 3 was adjusted for variables in model 2 plus antidiabetic drugs, lipid lowering drugs and antihypertensive drugs. Model 4 was adjusted for variables in model 3 plus diabetic nephropathy. Model 5 was adjusted for variables in model 4 and Tyr or Phe respectively. The concentration of Phe was coded to $45.34 \mu \mathrm{mol} / \mathrm{L}$ (mean) if Phe $\geq 100.00 \mu \mathrm{mol} / \mathrm{L}$. Missing values of glycated hemoglobin and lipids were presented as one category.

DR, diabetic retinopathy; Phe, phenylalanine; T2D, type 2 diabetes; Tyr, tyrosine.

is $\geq 100.00 \mu \mathrm{mol} / \mathrm{L}$. Missing values of HbAlc and lipids were presented as one category.

\section{Potential increase in predictive values of tyrosine and phenylalanine for DR}

Inclusion of tyrosine or/and phenylalanine in the traditional risk factor model significantly increased the area under the curve (AUC) from 0.81 to $0.83(\mathrm{p}<0.05)$ (figure 2$)$.

\section{Additive interactions between pairs of tyrosine and phenylalanine and DN status for DR}

Using copresence of tyrosine $\geq 64 \mu \mathrm{mol} / \mathrm{L}$ and phenylalanine $\geq 64 \mu \mathrm{mol} / \mathrm{L}$ as the reference, tyrosine $<64 \mu \mathrm{mol} / \mathrm{L}$ alone or phenylalanine $<64 \mu \mathrm{mol} / \mathrm{L}$ alone was not associated with increased risk of DR, while copresence of tyrosine $<64 \mu \mathrm{mol} / \mathrm{L}$ and phenylalanine $<64 \mu \mathrm{mol} / \mathrm{L}$ was associated with a markedly increased risk of DR (OR 6.01,

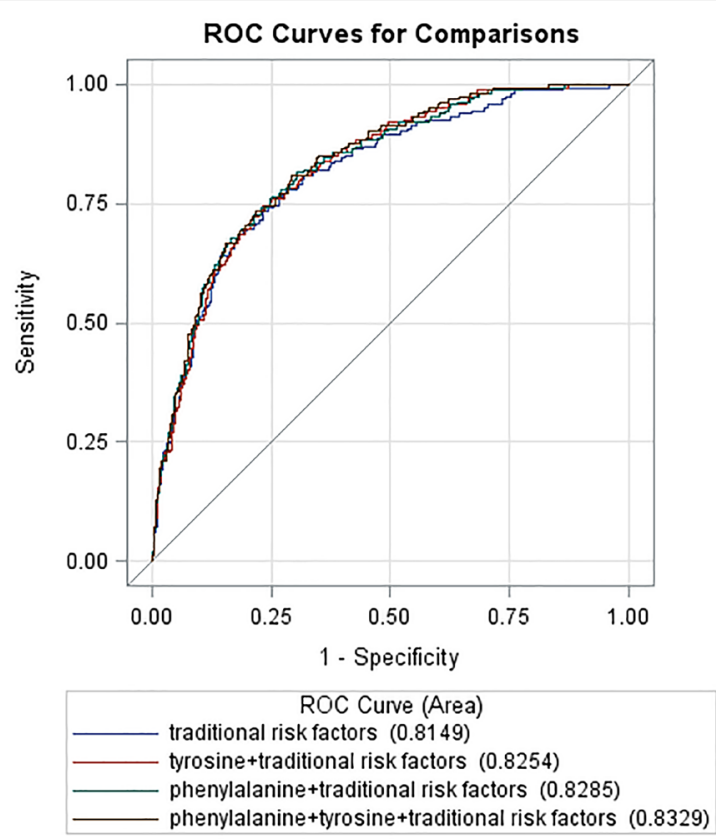

Figure 2 ROC curves of traditional risk factors and traditional risk factors plus amino acids for diabetic retinopathy in type 2 diabetes. The blue curve stands for the traditional risk factor model (multivariable model 1 in table 2 for the list of variables); the red curve stands for the traditional risk factors plus the tyrosine model; the green curve stands for the traditional risk factors plus the phenylalanine model; the black curve stands for the traditional risk factors plus the phenylalanine and tyrosine models. The area under the operating characteristic curve was $0.81(95 \% \mathrm{Cl} 0.78$ to 0.85$)$ for the traditional risk factor model, $0.83(95 \% \mathrm{Cl} 0.79$ to 0.86$)$ for the traditional risk factor plus tyrosine model, $0.83(95 \% \mathrm{Cl} 0.80$ to 0.86$)$ for the traditional risk factor plus phenylalanine model, $0.83(95 \% \mathrm{Cl}$ 0.80 to 0.86 ) for the traditional risk factor plus phenylalanine and tyrosine model $(p<0.05$ for comparison of the traditional risk factor model with the rest of the three models). ROC, receiver operating characteristic.

95\% CI 1.35 to 26.8 ), with a significant additive interaction (AP $0.81,95 \%$ CI 0.42 to 1.20 ; RERI $4.86,95 \%$ CI -1.27 to 10.98 ; S $32.5,95 \%$ CI 6.94 to $15.2 \mathrm{e}^{10}$ ) (table 3 ).

There was a significant additive interaction between DN and copresence of tyrosine $<64 \mu \mathrm{mol} / \mathrm{L}$ and phenylalanine $<64 \mu \mathrm{mol} / \mathrm{L}$ for DR risk. Patients with DN but without copresence of tyrosine $<64 \mu \mathrm{mol} / \mathrm{L}$ and phenylalanine $<64 \mu \mathrm{mol} / \mathrm{L}$ had a 5.7-fold risk of DR compared with patients without exposures to any of the two risk factors (OR 5.65, 95\% CI 1.20 to 26.50). Similarly, copresence of tyrosine $<64 \mu \mathrm{mol} / \mathrm{L}$ and phenylalanine $<64 \mu \mathrm{mol} / \mathrm{L}$ alone was associated with a 6.7-fold risk of DR (OR 6.68, $95 \%$ CI 2.36 to 18.9). Exposures to both risk factors markedly increased the ORs of exposure to any one of them alone to 25.9 (95\% CI 8.71 to 76.9 ). The additive interaction measures were highly significant (AP $0.56,95 \%$ CI 0.25 to 0.88 ; RERI $14.6,95 \%$ CI -3.84 to 33.0 ; and S 2.41, 95\% CI 1.10 to 5.27) (table 3). 
Table 3 Additive interaction of Tyr, Phe and DN for DR in type 2 diabetes

\begin{tabular}{|c|c|c|c|}
\hline & OR & $95 \% \mathrm{Cl}$ & $P$ value \\
\hline \multicolumn{4}{|l|}{ Tyr and Phe ( $\mu \mathrm{mol} / \mathrm{L})$} \\
\hline $\begin{array}{l}\text { Tyr } \geq 64 \text { and } \\
\text { Phe } \geq 64\end{array}$ & Reference & & \\
\hline $\begin{array}{l}\text { Tyr }<64 \text { and } \\
\text { Phe } \geq 64\end{array}$ & 0.64 & 0.08 to 5.05 & 0.671 \\
\hline $\begin{array}{l}\text { Tyr } \geq 64 \text { and } \\
\text { Phe }<64\end{array}$ & 1.52 & 0.25 to 9.26 & 0.652 \\
\hline $\begin{array}{l}\text { Tyr }<64 \text { and } \\
\text { Phe }<64\end{array}$ & 6.01 & 1.35 to 26.8 & 0.019 \\
\hline \multicolumn{4}{|l|}{ Measure } \\
\hline RERI & 4.85 & -1.27 to 11.0 & \\
\hline AP & 0.81 & 0.42 to 1.20 & \\
\hline$S$ & 32.5 & 6.94 to $15.2 \mathrm{e}^{10}$ & \\
\hline \multicolumn{4}{|c|}{ Tyr, Phe and DN ( $\mu \mathrm{mol} / \mathrm{L})$} \\
\hline $\begin{array}{l}\text { Tyr } \geq 64 \text { or } \\
\text { Phe } \geq 64 \text { and non- } \\
\text { DN }\end{array}$ & Reference & & \\
\hline $\begin{array}{l}\text { Tyr } \geq 64 \text { or } \\
\text { Phe } \geq 64 \text { and DN }\end{array}$ & 5.65 & 1.20 to 26.5 & 0.028 \\
\hline $\begin{array}{l}\text { Tyr }<64 \text { and } \\
\text { Phe }<64 \text { and non- } \\
\text { DN }\end{array}$ & 6.68 & 2.36 to 18.9 & $<0.001$ \\
\hline $\begin{array}{l}\text { Tyr }<64 \text { and } \\
\text { Phe }<64 \text { and DN }\end{array}$ & 25.9 & 8.71 to 76.9 & $<0.000$ \\
\hline \multicolumn{4}{|l|}{ Measure } \\
\hline RERI & 14.6 & -3.84 to 33.0 & \\
\hline AP & 0.56 & 0.25 to 0.88 & \\
\hline$S$ & 2.41 & 1.10 to 5.27 & \\
\hline
\end{tabular}

Adjusted for age, sex, body mass index, duration of diabetes, systolic blood pressure, glycated hemoglobin, low-density lipoprotein cholesterol, high-density lipoprotein cholesterol, triglyceride, DN, antidiabetic drugs, lipid-lowering drugs and antihypertensive drugs. Significant RERI $>0, A P$ due to interaction $>0$ or $S>1$ indicate a significant additive interaction.

$\mathrm{AP}$, attributable proportion; DN, diabetic nephropathy; DR, diabetic retinopathy; Phe, phenylalanine; RERI, relative excess risk due to interaction; S, synergy index; Tyr, tyrosine.

\section{Global amino acid profile according to DR status}

Asparagine, glutamate, glycine, histidine, leucine, methionine, serine, tryptophan and valine in the DR group were significantly lower than those in the non-DR group. Alanine, arginine, aspartic acid, citrulline, cystine, glutamine, homocysteine, lysine, ornithine, proline and threonine were similar between the two groups (online supplementary table S1).

\section{Characteristics and associations of tyrosine and phenylalanine with DR risk in the subgroup from propensity score matching}

A total of 162 pairs individuals were matched. Duration of diabetes, use of insulin, HbAlc and other factors except for TG were at similar levels in the two groups (online supplementary table S2).

Tyrosine and phenylalanine were significantly lower in the retinopathy group (online supplementary table
S2). The ORs of tyrosine and phenylalanine for DR were significant in both univariable and multivariable analyses. The effect size was larger in the subgroup than in the total cohort (multivariable analysis: OR for tyrosine: 4.26, 95\% CI 1.59 to 11.4; OR for phenylalanine: $8.06,95 \%$ CI 2.58 to 25.1 ) (online supplementary table S3).

\section{DISCUSSION}

Using this cross-sectional study, we detected an interaction between plasma tyrosine and phenylalanine for DR risk in T2D. The increased DR risk was observed only in patients with both abnormalities, that is, low tyrosine and low phenylalanine, suggesting that the effect of low tyrosine (phenylalanine) on DR was conditional on the presence of each other. Furthermore, we found a significant interaction between DN and coexistence of low tyrosine and low phenylalanine. DR risk was increased in patients with exposure to either of the risk factors and especially in patients with both DN and low tyrosine plus low phenylalanine levels, suggesting that control of tyrosine/ phenylalanine metabolism might confer great risk reduction for DR in patients with T2D with and without DN.

Compared with glucose and lipid metabolism, amino acid metabolism drew less but emerging concern for metabolic disorders. In this regard, it was possible to discover a new target from this point of view. We found in our study that tyrosine and phenylalanine were inversely associated with DR risk. Consistently, a case-cohort study reported that higher tyrosine was associated with decreased risk of microvascular disease composed of DN and DR. However, no association was found between phenylalanine and microvascular disease in that investigation. ${ }^{10}$ The difference might be due to different outcomes (DR and DN in this cohort, whereas DR in our study) or ethnicity or study design.

In our analysis, effects of tyrosine and phenylalanine on DR were contingent on the presence of the other risk factor. Only patients with both exposures, that is, low tyrosine and low phenylalanine, were at higher risk of DR. As stated previously, tyrosine and phenylalanine were the preferred and secondary precursors of $\mathrm{DA}^{7}$ DA dysregulation contributed to retinal neurodegeneration and treatment with L-DOPA preserved partial visual function. ${ }^{56}$ Probably, in patients with low tyrosine and low phenylalanine, substrate of DA synthesis was insufficient; in patients with either high phenylalanine or tyrosine, DA synthesis was not blocked. ${ }^{7}$ Apart from production, catecholamine release was also influenced by tyrosine concentration; that is, higher blood tyrosine accelerated catecholamine release. ${ }^{22}$ It was interesting to note that although subjects without T2D were with lower blood tyrosine or phenylalanine according to previous research, ${ }^{1423}$ risk of renal injury was lower than in patients with T2D. Possibly, the effect of tyrosine and phenylalanine on renal damage was based on other diabetesrelated conditions; that is, DA deficiency was a kind of relative insufficiency. Nishimura and Kuriyama ${ }^{24}$ found 
that rats with and without hyperglycemia had no difference in DA production, but a significantly accelerated depletion of DA was found in diabetic rats, suggesting more substrate requirement in diabetes, which supported our finding. Protein content of diet was proved to modify tyrosine concentration, and the elevated retinal tyrosine concentration stimulated L-DOPA synthesis in the light but not in the dark. ${ }^{25}$ Taken together, our study probably suggested that appropriate protein diet and light could assist DR treatment.

We found that pure lack of tyrosine and phenylalanine increased DR risk probably by DA deficiency, whereas copresence with DN greatly accelerated DR development by an unidentified mechanism. DN itself was a composite manifestation of a series of risk factors ${ }^{26}$ and represented severe endothelium impairment. ${ }^{11}$ Our analysis might suggest an interaction of risk factors or endothelium impairment and neurodegeneration on DR. Indeed, previous studies had reported a vicious cycle between microvascular impairment and neurodegeneration. ${ }^{47}$ In short, neurodegeneration participated in blood-retinal barrier breakdown, vasoconstriction, and impaired neurovascular coupling, which aggravated the microvascular impairment. Microvascular impairment, in turn, accelerated toxicity of toxic substances to neurodegeneration. Our research generated new hypotheses for basic scientific researchers to investigate a possible pathway that links neurodegeneration to microvascular impairment.

Our findings had important clinical implications: (1) since introducing tyrosine and phenylalanine into the model increased AUC, tyrosine and phenylalanine may be candidate markers for predicting DR in T2D; (2) in patients with diabetes without $\mathrm{DN}$, clinicians might pay attention to abnormal tyrosine/phenylalanine metabolism and neurodegeneration; (3) given the interaction of amino acids metabolism and DN, it is plausible that regulation of tyrosine/phenylalanine metabolism in patients with diabetes with DN or improvement of renal function in patients with abnormal tyrosine/phenylalanine metabolism may reduce much DR risk in patients with T2D. Besides, Asian patients with T2D were more likely to suffer $\mathrm{DN},{ }^{28}$ so our finding may benefit Asians more than the white race; and (4) since neurodegeneration tends to come earlier than other detectable signs, our findings may establish an early treatment window for DR therapy.

Our study also has limitations. First, our study was a retrospective cross-sectional survey, so the causality could not be verified. However, our findings were consistent with previous studies, which to some extent supported our results. Second, the nature of a cross-sectional study prevented us from establishing any causal relationships of low tyrosine and phenylalanine with retinopathy. We cannot exclude the possibility that low tyrosine and phenylalanine resulted from metabolic abnormalities that included but were not limited to insulin resistance and insulin insufficiency. However, the sensitivity analysis showed that after adjustment for traditional risk factors such as BMI, hyperglycemia and use of insulin, the ORs became numerically larger, which seems not to support a reverse association. These findings need to be further tested in prospective cohort studies. Third, there were lots of missing values in HbAlc, HDL-C, LDL-C and TGs. Given that glucose and lipids were not our primary research purposes, to avoid great information lack, we recognized missing values as one category. Fourth, we did not adjust for eGFR and albuminuria in multivariable models. eGFR and albuminuria were typically symptoms of DN and we adjusted for DN instead. Fifth, we did not collect information on lifestyle. Sixth, we did not collect information on DA concentration.

In conclusion, our study detected inverse associations of plasma levels of tyrosine and phenylalanine with risk of DR in Chinese patients with T2D. Low tyrosine and low phenylalanine levels had a significant additive interaction toward increasing the risk of DR, which was further amplified by the presence of DN. Our findings support that there were complicated interplays among tyrosine, phenylalanine and DN. Further prospective cohort studies are warranted to confirm these findings in Chinese and non-Chinese populations with T2D mellitus. Mechanistic investigations are also needed to better understand the role of disturbance in tyrosine and phenylalanine metabolism, especially under the circumstance of $\mathrm{DN}$.

\section{Author affiliations}

${ }^{1}$ Department of Toxicology and Sanitary Chemistry, School of Public Health, Tianjin Medical University, Tianjin, China

${ }^{2}$ Chinese Academy of Sciences, Dalian Institute of Chemical Physics, Dalian, China ${ }^{3}$ Department of Radiotherapy Oncology, The Second Affiliated Hospital of Dalian Medical University, Dalian Medical University, Dalian, China

${ }^{4}$ Department of Epidemiology and Biostatistics, School of Public Health, Tianjin Medical University, Tianjin, China

${ }^{5}$ Tianjin Key Laboratory of Environment, Nutrition and Public Health, Tianjin, China

Acknowledgements The authors thank all the physicians and doctors in the Second Affiliated Hospital of Dalian Medical University who participated in the study and extended their support for data collection and also all the laboratory technicians in Dalian Institute of Chemical Physics for measuring the metabolites.

Contributors Z-ZF and XY designed the study. X-FF and JuL collected the data. $\mathrm{H}-\mathrm{HL}$ analyzed the data and wrote the draft. JiL and X-YS gave critical comments and contributed to the writing of this article.

Funding This work was supported by the project for the National Key R\&D Program of China (2019YFA0802300 and 2019YFA0802302), the 13th Five Year Plan and TMU Talent project (11601501/2016KJ0313), and the Open Project of the Key Laboratory of Modern Toxicology of Ministry of Education, Nanjing Medical University (NMUMT201809), United Funding of Individualized diagnosis and treatment collaborative center, and the Open Project of Guangdong Key Laboratory of nutrition, diet and health (2019-GLFNH-02).

Competing interests None declared.

Patient consent for publication Not required.

Ethics approval The Ethics Committee for Clinical Research of FAHLMU approved the ethics of the study (reference number 2015-02), and informed consent was waivered due to the retrospective nature of the study, which is consistent with the Declaration of Helsinki.

Provenance and peer review Not commissioned; externally peer reviewed.

Data availability statement Data are available upon reasonable request. Our study collected clinical information from electronic medical system retrospectively. Our data are not in a repository. If someone is interested in our data, please contact us via email (fangzhongze@tmu.edu.cn). One should include his detailed statistical analysis plans in his email. 
Open access This is an open access article distributed in accordance with the Creative Commons Attribution Non Commercial (CC BY-NC 4.0) license, which permits others to distribute, remix, adapt, build upon this work non-commercially, and license their derivative works on different terms, provided the original work is properly cited, appropriate credit is given, any changes made indicated, and the use is non-commercial. See: http://creativecommons.org/licenses/by-nc/4.0/.

ORCID iD

Zhong-Ze Fang http://orcid.org/0000-0003-1113-9262

\section{REFERENCES}

1 Sabanayagam C, Chee ML, Banu R, et al. Association of diabetic retinopathy and diabetic kidney disease with all-cause and cardiovascular mortality in a multiethnic Asian population. JAMA Netw Open 2019;2:e191540.

2 Wang FH, Liang YB, Zhang F, et al. Prevalence of diabetic retinopathy in rural China: the Handan eye study. Ophthalmology 2009;116:461-7.

3 Mohamed Q, Gillies MC, Wong TY. Management of diabetic retinopathy: a systematic review. JAMA 2007;298:902-16.

4 Simó R, Hernández C, European Consortium for the Early Treatment of Diabetic Retinopathy (EUROCONDOR). Neurodegeneration in the diabetic eye: new insights and therapeutic perspectives. Trends Endocrinol Metab 2014;25:23-33.

5 Kim MK, Aung MH, Mees L, et al. Dopamine deficiency mediates early Rod-Driven inner retinal dysfunction in diabetic mice. Invest Ophthalmol Vis Sci 2018:59:572-81.

6 Aung MH, Park HN, Han MK, et al. Dopamine deficiency contributes to early visual dysfunction in a rodent model of type 1 diabetes. $J$ Neurosci 2014;34:726-36.

7 Fernstrom JD, Fernstrom $\mathrm{MH}$. Tyrosine, phenylalanine, and catecholamine synthesis and function in the brain. J Nutr 2007;137:1539S-47. discussion 48S.

8 Adams SH. Emerging perspectives on essential amino acid metabolism in obesity and the insulin-resistant state. Adv Nutr 2011;2:445-56.

9 Wurtman RJ, Larin F, Mostafapour S, et al. Brain catechol synthesis: control by train tyrosine concentration. Science 1974;185:183-4

10 Welsh P, Rankin N, Li Q, et al. Circulating amino acids and the risk of macrovascular, microvascular and mortality outcomes in individuals with type 2 diabetes: results from the ADVANCE trial. Diabetologia 2018;61:1581-91.

11 Satchell SC, Tooke JE. What is the mechanism of microalbuminuria in diabetes: a role for the glomerular endothelium? Diabetologia 2008;51:714-25.

12 Wong CW, Wong TY, Cheng C-Y, et al. Kidney and eye diseases: common risk factors, etiological mechanisms, and pathways. Kidney Int 2014;85:1290-302.
13 Kopple JD. Phenylalanine and tyrosine metabolism in chronic kidney failure. J Nutr 2007;137:1586S-90. discussion 97S-98S.

$14 \mathrm{Li} \mathrm{J}$, Cao Y-F, Sun X-Y, et al. Plasma tyrosine and its interaction with low high-density lipoprotein cholesterol and the risk of type 2 diabetes mellitus in Chinese. J Diabetes Investig 2019;10:491-8.

15 Alberti KG, Zimmet PZ. Definition, diagnosis and classification of diabetes mellitus and its complications. Part 1: diagnosis and classification of diabetes mellitus provisional report of a who consultation. Diabet Med 1998;15:539-53.

16 WPRO. The Asia Pacific perspective: redefining obesity and its treatment.

17 American Diabetes Association. Standards of Medical Care in Diabetes-2019 Abridged for Primary Care Providers. Clin Diabetes 2019;37:11-34

18 Huang Q, Sun X, Chen Y, et al. A study of the applicability of GFR evaluation equations for an elderly Chinese population. J Nutr Health Aging 2015;19:693-701.

19 Wang Q, Sun T, Cao Y, et al. A dried blood spot mass spectrometry metabolomic approach for rapid breast cancer detection. Onco Targets Ther 2016;9:1389-98.

20 May M. Regression Modelling Strategies with Applications to Linear Models, Logistic Regression, and Survival Analysis. Frank E Harrell Jr, New York: Springer 2001, pp. 568, \$79.95. ISBN 0-387-95232-2. Int J Epidemiol 2002;31:699-700.

21 Li J, Huo X, Cao Y-F, et al. Bile acid metabolites in early pregnancy and risk of gestational diabetes in Chinese women: a nested casecontrol study. EBioMedicine 2018;35:317-24.

22 Le Masurier M, Oldenzeil W, Lehman C, et al. Effect of acute tyrosine depletion in using a branched chain amino-acid mixture on dopamine neurotransmission in the rat brain. Neuropsychopharmacology 2006;31:310-7.

23 Merino J, Leong A, Liu C-T, et al. Metabolomics insights into early type 2 diabetes pathogenesis and detection in individuals with normal fasting glucose. Diabetologia 2018;61:1315-24.

24 Nishimura C, Kuriyama K. Alterations in the retinal dopaminergic neuronal system in rats with streptozotocin-induced diabetes. $J$ Neurochem 1985;45:448-55.

25 Fernstrom MH, Fernstrom JD. Protein consumption increases tyrosine concentration and in vivo tyrosine hydroxylation rate in the light-adapted rat retina. Brain Res 1987;401:392-6.

26 Yang X, Ko GTC, So WY, et al. Additive interaction of hyperglycemia and albuminuria on risk of ischemic stroke in type 2 diabetes: Hong Kong diabetes registry. Diabetes Care 2008;31:2294-300.

27 Penno G, Solini A, Zoppini G, et al. Rate and determinants of association between advanced retinopathy and chronic kidney disease in patients with type 2 diabetes: the renal insufficiency and cardiovascular events (RIACE) Italian multicenter study. Diabetes Care 2012;35:2317-23.

28 Pan CY, Ho LT, Soegondo S, et al. Prevalence of albuminuria and cardiovascular risk profile in a referred cohort of patients with type 2 diabetes: an Asian perspective. Diabetes Technol Ther 2008;10:397-403. 